Abstract 380 Table 2 Comparision of fatigue between three cohorts ${ }^{\mathrm{a}}$ of inflamatory disorder with disease duration of less than one year.

\begin{tabular}{|c|c|c|c|c|c|c|}
\hline & $\operatorname{SLE}(n=70)$ & $\mathrm{SSc}(\mathrm{n}=74)$ & AAV $(n=67)$ & & p-value & \\
\hline & Median (IQR) & Median (IQR) & Median (IQR) & $\begin{array}{c}\text { SLE } \\
\text { vs } \\
\text { SSc }\end{array}$ & $\begin{array}{c}\text { SLE } \\
\text { vs } \\
\text { AAV }\end{array}$ & $\begin{array}{l}\text { SSc vs } \\
\text { AAV }\end{array}$ \\
\hline Age (years) & $37.5(28.48 .5)$ & $62.5(49.75-69)$ & $59(46-69)$ & $<0.001$ & $<0.001$ & 0.666 \\
\hline \multicolumn{7}{|l|}{ Fatigue } \\
\hline Global' $(1-50)$ & $36.7(24.4-42.2)$ & $23.9(11.8-34.9)$ & $30.3(20.0-37.1)$ & $<0.001$ & 0.015 & 0.049 \\
\hline Degree $^{c}(1-10)$ & $8(6-9)$ & $6(3-8)$ & $7(5-9)$ & $<0.001$ & 0.030 & 0.013 \\
\hline Severity $^{c}(1-10)$ & $8(5-9)$ & $5(2-7)$ & $7(5-8)$ & $<0.001$ & 0.071 & 0.006 \\
\hline Distress $^{\mathrm{c}}(1-10)$ & $7(3-8)$ & $4(1-7)$ & $6(3-8)$ & $<0.001$ & 0.172 & 0.009 \\
\hline Activities $^{c}(1-10)$ & $4.7(2.5-6.6)$ & $2.9(1.2-5.5)$ & $3.2(1.9-5.1)$ & 0.003 & 0.016 & 0.514 \\
\hline Anxiety $^{d}(0-14)$ & $6(2-11)$ & $4(2-6)$ & $7.0(3-10)$ & 0.020 & 0.866 & 0.011 \\
\hline Depression ${ }^{d}(0-14)$ & $5(1-8.3)$ & $3(1-6)$ & $4.0(2-8)$ & 0.084 & 0.779 & 0.221 \\
\hline \multicolumn{7}{|c|}{$\begin{array}{l}\text { a: } S L E=\text { systemic iupus erythematosus, } S S=\text { systemic sclerosis, } A A V=\text { and anti-neutrophil cytoplasmatic antibody } \\
\text { associated vasculitis }\end{array}$} \\
\hline \multicolumn{7}{|l|}{ b:Mann_Whitney $U$} \\
\hline \multicolumn{7}{|c|}{ c: Fatigue components from Multi Assessment of Fatigue Scale } \\
\hline
\end{tabular}

(Hospital Anxiety and Depression Scale). Sub-analyses of MAF were conducted of global fatigue (scale 1-50) and subscales regarding degree, severity, distress and impact on daily activities (scale 1-10 for each). Results are presented as median (inter-quartile range).

Results We included 857 patients (77\% women), age 18-85 years, disease duration 0-58 years. Persons with SLE were younger 47(34-57) than persons with SSc 60(52-69) $\mathrm{p}<0.001$ and AAV 62(49-69) $\mathrm{p}<0.001$, and they had longer disease duration $10(2-20)$ than both the SSc $2(0-8)$ p $<0.001$ and the AAV $3(0-8)$ p $<0.001$ groups.

SLE patients reported a higher anxiety level and more impact of fatigue on all analysed subscales compared to the AAV patients, and all but impact on daily activities compared to SSc. SSc and AAV had a similar pattern on all analysed components (Table 1).

Among persons with disease duration less than a year, SLE still scored highest on all components of fatigue, but interestingly AAV patients reported similar figures to SLE regarding the severity and distress of fatigue (Table 2).

Conclusions Fatigue and anxiety was assessed to be more pronounced and have more impact on daily actives in SLE patients compared to SSc and AAV patients. However in persons with short disease duration the pattern of fatigue in SLE was more similar to AAV than SSs.

\section{LABORATORY FINDINGS OF SYNOVIAL FLUID IN CONNECTIVE TISSUE DISEASES}

V Peycheva*. University hospital "St. Ivan Rilski", Rheumatology, Sofia, Bulgaria

\subsection{6/lupus-2017-000215.381}

Background and aims Changes in the parameters of synovial fluid (SF) give a picture of the local pathological process in connective tissue diseases. 
To investigate different parameters in SF of patients with systemic lupus erythematodes (SLE), rheumatoid arthritis (RA) and osteoarthritis (OA).

Methods We describe the evaluation of SF in 28 SLE, 41 RA, and 36 OA patients. SF is collected via arthrocentesis in heparinized or EDTA tubes. The diagnosis was established in all subjects prior to SF examination based on typical clinical and laboratory features. The clinical activity of the diseases at the time of joint aspiration varied.

Results The white blood cell (WBC) count in 28 SLE patients, ranging from 500 to 12250 with an average count of 3473 cells $/ \mu$ with $55 \%$ polymorphic nuclear cells (PMNs), was significantly lower than in RA - 11048 cells/ $\mu$ l with $75 \%$ PMNs. The WBC count in OA patients was significantly lower - $3718 \pm 2373$ cells $/ \mu$ l. The highest protein levels were found in RA patients, followed by SLE and OA patients: total protein respectively $50.3 \pm 6.9$ vs $45 \pm 7.3$ vs $48.6 \pm 10.9 \mathrm{~g} / \mathrm{L}$ and IgG concentration - $21.22 \pm 3.53$ vs $9.53 \pm 4.27$ vs $18 \pm 2.48 \mathrm{~g} /$ L. Circulating Immune Complexes were significantly higher in the RA group compared to SLE group and OA: 0.247 \pm 0.07 vs $0.193 \pm 0.05$ vs $0.108 \pm 0.40 \mathrm{mg} / \mathrm{ml}$.

Conclusions The analysis of the SF of lupus patients has shown elevated levels of WBCs, total protein and circulating immune complexes as a markers for the high SLE activity. Synovial fluid is a possibility to define the type of arthritis in different rheumatic diseases.

\section{ANA AND ENA TESTING ALGORITHM: PERSPECTIVE OF A LARGE PUBLIC HOSPITAL LABORATORY IN NEW ZEALAND}

K Smith*. North Shore Hospital, Special Assays, Auckland, New Zealand

\subsection{6/lupus-2017-000215.382}

Background and aims An ANA and ENA testing algorithm was established at the immunology laboratory at Waitemata District Health Board (WDHB) in New Zealand. WDHB serves more than 500000 people in central New Zealand. Due to the high demand for ANA testing, WDHB opted for an automated ANA EIA screening and Extractable Nuclear Antibodies (ENA) EIA reflex testing algorithm, with HEp-2 IFA as an option when there is a strong indication of false negative results.

Methods From January 2012 to April 2016, 8515 patient samples were tested with ANA Screening EIA kits, and 1624 samples were reflex tested with ENA EIA kits for detection of antibodies to SSA, SSB, Sm, Sm/RNP, Scl-70, Jo-1, dsDNA and centromere (Bio-Rad Laboratories, California, USA). The reflex testing is triggered when either the screening result was positive or requested by a clinician. ANA IFA tests were performed on request.

Results The general ANA screening positive rate was $18.5 \%$ $(1585 / 8515)$ in the WDHB population. The positivity rate for each individual ENA is shown in Table 1 . The overall positive rate for ENA testing was 54.8\% (890/1624) indicating that the ANA screening has been effective in detecting the specific presence of ENAs.

Conclusions Using this ANA and ENA testing algorithm, WDHB was able to screen a large number of patient samples and quickly identify specific ENA all in one day, resulting in improved workflow and significant labour and cost savings.

\section{PLASMA ADAMTS-13 ACTIVITY IN PROLIFERATIVE LUPUS NEPHRITIS: A LARGE COHORT STUDY FROM CHINA}

${ }^{1} Y$ Tan. ${ }^{1}$ Peking University First Hospital, Nephrology Department, Bei Jing, China

\subsection{6/lupus-2017-000215.383}

Background and aims The aim of this study was to investigate plasma ADAMTS-13 activity in proliferative lupus nephritis patients, and evaluate their correlations with clinical, laboratory and pathological features, especially the vascular lesions in lupus nephritis.

Methods Plasma samples from 163 biopsy-proven class III and IV lupus nephritis patients and 98 normal controls were collected. ADAMTS-13 activity was evaluated by residual collagen binding assay. IgG autoantibodies against ADAMTS-13 were detected by ELISA. Levels of vWF were evaluated by ELISA. Their associations with clinical, laboratory and pathological features were further assessed.

Results Plasma ADAMTS-13 activity in lupus nephritis patients was significantly lower than that in normal controls $(84 \%$ $\pm 21 \% \%$ vs. $90 \% \pm 13 \%, \mathrm{p}=0.005)$. The plasma levels of vWF was significantly higher in lupus nephritis group than that in normal controls $(1.00 \pm 0.79$ vs. $0.70 \pm 0.30, p=0.025)$. Plamsa ADAMTS-13 activity was negatively correlated with the level of serum creatinine and proteinuria $(\mathrm{r}=-0.354, \mathrm{p}<0.001$; $r=-0.200, p=0.011$, respectively). Patients with higher ADAMTS-13 activity had significantly higher levels of factor $\mathrm{H} \quad(401.51 \pm 183.01 \mu \mathrm{g} / \mathrm{ml} \quad$ vs. $239.02 \pm 155.45 \mu \mathrm{g} / \mathrm{ml}$, $\mathrm{p}=0.005)$. Plasma ADAMTS-13 activity was negatively associated with the total pathological AI scores, acute glomerular vascular lesions, acute renal vascular lesions (all $p<0.001$ ) and tubular atrophy $(\mathrm{p}=0.011)$. Low activity of ADAMTS-13 was a risk factor for renal outcomes $(\mathrm{p}=0.039, \mathrm{HR}=0.047$, 95\% CI: 0.120-1.005).

Conclusions Decreased ADAMTS-13 activity was found in proliferative lupus nephritis patients and plasma ADAMTS-13 activity was closely associated with renal injury indices, especially pathological vascular scores. The role of ADAMTS-13 in the disease need to be further investigated.

\section{MULTI-SPECIALISTS' PERSPECTIVES ON CLINICAL DECISION MAKING IN SYSTEMIC LUPUS ERYTHEMATOSUS: AN INTERVIEW STUDY}

${ }^{1,2} \mathrm{D}$ Tunnicliffe ${ }^{*},{ }^{3,4,5} \mathrm{D}$ Singh-Grewal, ${ }^{1,2} \mathrm{JC}$ Craig, ${ }^{6} \mathrm{~S}$ Jesudason, ${ }^{3,7} \mathrm{M}$.W. Lin, ${ }^{4,8} \mathrm{~S} \mathrm{O}^{\prime} \mathrm{Neill}_{1}$ ${ }^{1,2,8} \mathrm{D}$ Sumpton, ${ }^{1,2} \mathrm{~A}$ Tong. ${ }^{1}$ University of Sydney, Sydney School of Public Health, Sydney, Australia; ${ }^{2}$ Children's Hospital at Westmead, Centre for Kidney Research, Sydney, Australia; ${ }^{3}$ The University of Sydney, Sydney Medical School, Sydney, Australia; ${ }^{4}$ The University of New South Wales, Faculty of Medicine, Sydney, Australia; ${ }^{5}$ The Sydney Children's Hospital Network, Department of Rheumatology, Sydney, Australia; ${ }^{6}$ Royal Adelaide Hospital, Central and Northern Adelaide Renal and Transplantation Service, Adelaide, Australia; 'Westmead Hospital, Department of Immunology, Sydney, Australia; ${ }^{8}$ Liverpool Hospital, Department of Rheumatology, Sydney, Australia

\subsection{6/lupus-2017-000215.384}

Background and aims Clinicians from different medical specialists are involved in the management of patients with systemic lupus erythematosus (SLE), however, unwarranted variation in practice remains largely unexplained. This study aims to describe specialists' attitudes and perspectives on the management of patients with SLE. 